

\title{
A Polyhedral Approach to Planar Augmentation and Related Problems*
}

\author{
Petra Mutzel \\ Max-Planck-Institut für Informatik, Im Stadtwald, \\ D-66123 Saarbrücken, Germany
}

\begin{abstract}
Given a planar graph $G$, the planar (biconnectivity) augmentation problem is to add the minimum number of edges to $G$ such that the resulting graph is still planar and biconnected. Given a nonplanar and biconnected graph, the maximum planar biconnected subgraph problem consists of removing the minimum number of edges so that planarity is achieved and biconnectivity is maintained. Both problems are important in Automatic Graph Drawing. In [JM95], the minimum planarizing $k$ augmentation problem has been introduced, that links the planarization step and the augmentation step together. Here, we are given a graph which is not necessarily planar and not necessarily $k$-connected, and we want to delete some set of edges $D$ and to add some set of edges $A$ such that $|D|+|A|$ is minimized and the resulting graph is planar, $k$-connected and spanning. For all three problems, we have given a polyhedral formulation by defining three different linear objective functions over the same polytope, namely the 2-node connected planar spanning subgraph polytope $2-\mathcal{N} \mathcal{C} \mathcal{P} \mathcal{L S}\left(K_{n}\right)$. We investigate the facial structure of this polytope for $k=2$, which we will make use of in a branch and cut algorithm. Here, we give the dimension of the planar, biconnected, spanning subgraph polytope for $G=K_{n}$ and we show that all facets of the planar subgraph polytope $\mathcal{P} \mathcal{L S}\left(K_{n}\right)$ are also facets of the new polytope $2-\mathcal{N} \mathcal{C} \mathcal{P} \mathcal{L} \mathcal{S}\left(K_{n}\right)$. Furthermore, we show that the node-cut constraints arising in the biconnectivity spanning subgraph polytope, are facet-defining inequalities for $2-\mathcal{N} \mathcal{C P} \mathcal{L} \mathcal{S}\left(K_{n}\right)$. We give first computational results for all three problems, the planar 2-augmentation problem, the minimum planarizing 2-augmentation problem and the maximum planar biconnected (spanning) subgraph problem. This is the first time that instances of any of these three problems can be solved to optimality.
\end{abstract}

\section{Introduction}

Many algorithms work only for biconnected graphs. A graph that does not satisfy this condition, has to be augmented by adding a set of edges such that the resulting graph is biconnected. In general, the problem of augmenting a graph by a minimum number of edges so that it meets certain edge or node connectivity requirements given by $k \in \mathrm{Z}$ is called augmentation problem. We will consider node connectivity requirements with $k=2$.

* Partially supported by DFG-Grant Ju204/7-1, Forschungsschwerpunkt “Effiziente Algorithme für diskrete Probleme und ihre Anwendungen" 
The (node-) connectivity $\kappa(G)$ of a graph $G$ is the minimum number of nodes whose removal together with its incident edges results in a disconnected or trivial graph. A graph is said to be $k$-node-connected, or $k$-connected, if $\kappa(G) \geq k$. The problem of finding the minimum cost $k$-node connected spanning subgraph in a general graph is proven to be NP-hard even for $k=2$ and uniform edge costs [GJ79]. Garg, Santosh and Singla have given a 3/2-approximation algorithm for $k=2$ and uniform edge costs [GSS93], whereas Ravi and Williamson have given a $2 \mathcal{H}(k)$-approximation algorithm for general $k$ and general costs, where $\mathcal{H}(k)=1+\frac{1}{2}+\ldots+\frac{1}{k}\left[\right.$ RW95]. For $G=K_{n}$, the complete graph on $n$ vertices, Harary has given a polynomial time algorithm for constructing a minimum $k$ vertex connected spanning subgraph for $k \in \mathrm{N}$ [Har62].

Another type of connectivity problems are augmentation problems. Here, the given graph has to be augmented in a minimum way to a $k$-connected graph. The problem of augmenting a given graph by the minimum number of edges in order to obtain a $k$-node connected graph seems still to be open, whereas there are polynomial time algorithms for $k=2$ and $k=3$ [RG77,HR91,WN93]. In the case that the augmented edges have general edge costs, the problem is NP-hard even for $k=2$ [ET76].

The planar ( $k$-)augmentation problem has been brought up by Kant, and consists of adding a minimum number of edges to a planar graph in order to obtain a $k$-connected graph, which is still planar. Kant showed that this problem is NP-hard for $k=2$, and gives a linear time approximation algorithm which adds at most 2 times the minimum required number of edges [Kan93].

The planar augmentation problem has a wide application in Automatic Graph Drawing. Here, many algorithms for drawing planar graphs work only for biconnected or even triconnected graphs. In order to use these algorithms for a simple connected graph there are two possibilities. Either to draw the biconnected parts of the graph separately, or to augment it to biconnectivity and draw the resulting graph while suppressing the augmented edges. In general, the second approach leads to nicer drawings.

Since there are many graph drawing algorithms for planar graphs and only a few algorithms for nonplanar graphs, Tamassia, Batini and Di Battista suggested a method using planarization [TBB88]. Here, the maximum planar subgraph $P$ of a given nonplanar graph is determined, and either $P$ is drawn and the deleted edges are inserted again, or the deleted edges are reinserted before the drawing step, and the produced crossings are substituted by artificial nodes in order to obtain a planar graph. In the former case, it is often required that the found maximum planar subgraph is biconnected. Requiring this condition and the condition that the subgraph should be spanning leads to the maximum planar biconnected (spanning) subgraph problem. Goldschmidt and Takvorian showed that even finding a biconnected spanning planar subgraph of a biconnected nonplanar graph is NP-hard [GT94].

For the application in Automatic Graph Drawing it is, indeed, advantageous to link the planarization step and the augmentation step together (see [JM95]). This leads to the minimum planarizing $k$-augmentation problem. Given a graph 
$G=(V, E)$, we like to delete a set of edges $D \subseteq E$ and to add a set of edges $A \subseteq(V \times V) \backslash E$ such that $|D|+|A|$ is minimum and the resulting graph is planar, spanning and $k$-connected.

All three problems, the planar $k$-augmentation problem, the maximum planar $k$-connected spanning subgraph problem and the minimum planarizing $k$ augmentation problem can be formulated as an optimization problem of a linear objective function over a single polytope. The planar $k$-connected (spanning) subgraph polytope $k-\mathcal{N} \mathcal{C P} \mathcal{L} \mathcal{S}\left(G_{0}\right)$ is defined to be the convex hull over all incidence vectors of planar, $k$-connected and spanning subgraphs of a graph $G_{0}$.

For a given graph $G=(V, E)$, we choose $G_{0}=\left(V_{0}, E_{0}\right)$ to be the complete graph on the vertex set $V_{0}=V$ and consider the optimization problem

$$
\max \left\{w^{T} x \mid x \in k-\mathcal{N} \mathcal{C P} \mathcal{L} \mathcal{S}\left(G_{0}\right)\right\}
$$

Depending on the value of the vector $w \in R^{E_{0}}$ we can formulate all three problems.

In order to solve the planar $k$-augmentation problem we set $w_{e}=-1$ for $e \in E_{0} \backslash E$ and $w_{e}=M$ for $e \in E$, where $M=\left|E_{0} \backslash E\right|+1$. By setting $w_{e}=1$ for $e \in E$ and $w_{e}=-M^{\prime}$ for $e \in E_{0} \backslash E$ with $M^{\prime}=|E|+1$, we can formulate the maximum planar $k$-connected spanning subgraph problem. The natural setting of $c$ occurs for the minimum planarizing $k$-augmentation problem. Here, $w_{e}=1$ for $e \in E$ and $w_{e}=-1$ for $e \in E_{0} \backslash E$. Maximizing $w^{T} x$ leads to taking as many edges of $G$ as possible and as few edges of $G_{0}-G$ as possible. This way, the difference between the given graph $G$ and the new graph will be minimized.

Hence, we are interested in the facial structure of the planar $k$-connected (spanning) subgraph polytope $k-\mathcal{N} \mathcal{C P} \mathcal{L} \mathcal{S}\left(K_{n}\right)$. The integer points of this polytope are identical to the integer points in the intersection of two well studied polytopes, namely the planar subgraph polytope $\mathcal{P} \mathcal{L} \mathcal{S}(G)$ for the maximum planar subgraph problem [JM95] and the $k$-node-connected spanning subgraph polytope $k-\mathcal{N} \mathcal{C S}(G)$ for the network-survivability problem [Sto92]. Since the polyhedral approach for both problems seems to be promising, i.e., the optimum solution can be found by branch and cut algorithms within a few seconds for graphs of moderate sizes, our hope is to obtain similar results for all three problems using the polyhedral approach.

The outline of the paper is as follows. In Section 2 we give some mathematical background in polyhedral combinatorics and recall the most important classes of facet-defining inequalities for the planar subgraph polytope. A short introduction into the $k$-connected subgraph polytope is given in Section 3. New results of the structure of the planar biconnected spanning subgraph polytope are contained in Section 4. Recall that we restrict our consideration to $k=2$, since many applications require biconnectivity. Moreover, in the second part of Section 4 we restrict ourselves to $k-\mathcal{N} \mathcal{C P} \mathcal{L S}\left(G_{0}\right)$ for $G_{0}=K_{n}$, the complete graph on $n$ vertices, which is not really a restriction, since all three problems can be formulated over $k-\mathcal{N} \mathcal{C P} \mathcal{L S}\left(K_{n}\right)$. In Section 5 we show how these theoretical results can be useful in practice. We describe our branch and cut algorithm which has been used in our computational experiments given in Section 6 . 


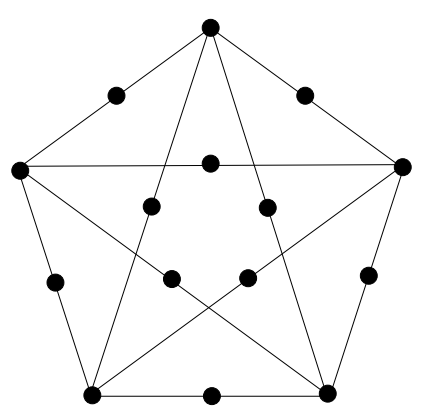

(a)

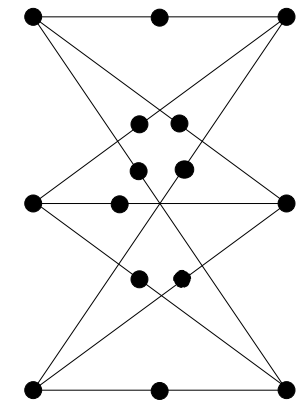

(b)

Fig. 1. shows some Kuratowski graphs, i.e., a subdivision of $K_{5}$ and $K_{33}$

\section{The Planar Subgraph Polytope}

Given a graph $G=(V, E)$ with edge weights $w_{e} \in \mathrm{R}$ for all $e \in E$, let $\mathcal{P}_{\mathcal{G}}$ be the set of all planar subgraphs of $G$. For each planar subgraph $P=\left(V^{\prime}, F\right) \in \mathcal{P}_{\mathcal{G}}$, we define its incidence vector $\chi^{F} \in \mathrm{R}^{E}$ by setting $\chi_{e}^{F}=1$ if $e \in F$ and $\chi_{e}^{F}=0$ if $e \notin F$. The planar subgraph polytope $\mathcal{P} \mathcal{L S}(G)$ of $G$ is defined as the convex hull over all incidence vectors of planar subgraphs of $G$. The problem of finding a planar subgraph $P=\left(V^{\prime}, F\right)$ of $G$ with weight $w(P)=\sum_{e \in F} w_{e}$ as large as possible can be written as the linear program $\max \left\{w^{T} x \mid x \in \mathcal{P} \mathcal{L} \mathcal{S}(G)\right\}$, since the vertices of the polytope $\mathcal{P} \mathcal{L} \mathcal{S}(G)$ are exactly the incidence vectors of the planar subgraphs of $G$. Kuratowski characterized the minimal nonplanar graphs to be exactly the subdivisions of $K_{5}$ and $K_{3,3}$. Hence we get the following integer programming formulation for the maximum planar subgraph problem:

$$
\begin{array}{ll}
\text { maximize } & w^{T} x \\
\text { subject to } & 0 \leq x_{e} \leq 1, \quad \text { for all } e \in E, \\
& x(K) \leq|K|-1, \text { for all Kuratoski subgraphs }\left(V^{\prime}, K\right), K \subseteq E \\
& x_{e} \text { integral, } \quad \text { for all } e \in E
\end{array}
$$

Since integer programming is NP-hard, we drop the integer constraints. In order to apply linear programming techniques to solve this linear program one has to represent $\mathcal{P} \mathcal{L S}(G)$ as the solution of an inequality system. Due to the NP-hardness of our problem, we cannot expect to be able to find a complete description of $\mathcal{P} \mathcal{L S}(G)$ by linear inequalities. But even a partial description of the facial structure of $\mathcal{P} \mathcal{L S}(G)$ by linear inequalities is useful for the design of a "branch and cut"-algorithm, because such a description defines a relaxation of the original problem. Such relaxations can be solved within a branch and bound framework via cutting plane techniques and linear programming in order to produce tight bounds. An irredundant description of $\mathcal{P} \mathcal{L S}(G)$ by linear inequalities contains only inequalities which describe proper faces of maximal dimension of $\mathcal{P} \mathcal{L S}(G)$, so-called facet-defining inequalities.

For efficiency, also in a partial description by inequalities, we concentrate on those valid inequalities for $\mathcal{P} \mathcal{L S}(G)$ which are facet-defining. For ease of notation, we define $x(F)=\sum_{e \in F} x_{e}$ for $F \subseteq E$. In [JM94] we state the following 


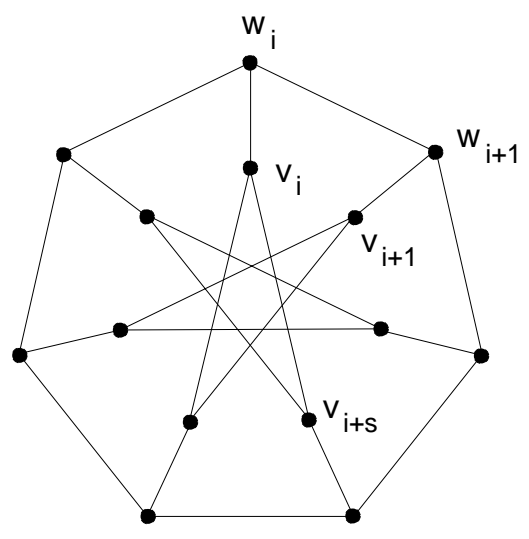

(a)

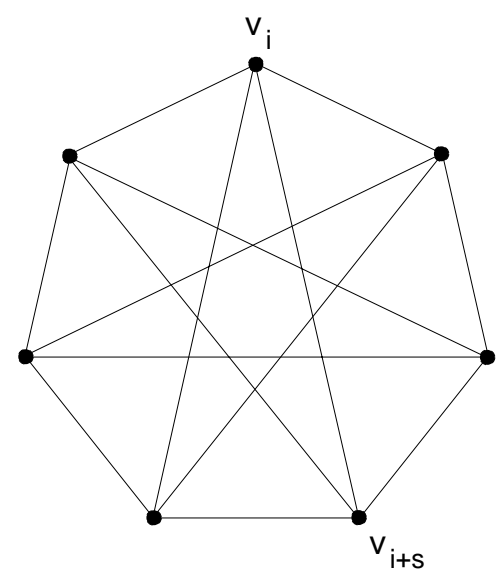

(b)

Fig. 2.(a) The generalized Petersen graph $P(7,3)$ and the (b) $s$-chorded cycle graph $G_{(n, s, t)}$ for $n=7$ and $s=3$

Theorem 1 [JM94]. The dimension of the planar subgraph polytope $\mathcal{P} \mathcal{L}(G)$ of $G=(V, E)$ is $|E|$, so it is full dimensional.

For all edges $e \in E$ the inequalities $x_{e} \geq 0$ and $x_{e} \leq 1$ define facets of $\mathcal{P} \mathcal{L}(G)$. For all subdivisions $G^{\prime}=\left(V^{\prime}, K\right)$ of $\bar{K}_{5}$ or $K_{3,3}$ contained in $G$ the inequality $x(K) \leq|K|-1$ defines a facet of $\mathcal{P} \mathcal{L} \mathcal{S}(G)$.

For all cliques $\left(V^{\prime}, F\right)$ (or complete bipartite subgraphs) contained in $G$, the Euler inequalities $x(F) \leq 3\left|V^{\prime}\right|-6$ (or $x(F) \leq 2\left|V^{\prime}\right|-4$, respectively) are facet-defining for $\mathcal{P} \mathcal{L S}(G)$.

The following class of graphs, called $s$-chorded cycle graphs, has been introduced in [Mut94b]. This class of graphs can be derived from the generalized Petersen graphs by the contraction of certain edges (see Figure 2). The $s$-chorded cycle graphs give rise to huge classes of inequalities generalizing the Kuratowski inequalities.

For the rest of this section all sums of integers representing nodes of graphs $G=(V, E)$, which are greater than $n=|V|$ are to be taken modulo $n$.

Definition 1 For $s, n, r, t \in \mathrm{N}, s \geq 2, n=s t+r, 0 \leq r<s$, the $s$-chorded cycle graph $G_{(n, s, t)}=(V, E)$ is defined via

$$
\begin{array}{lll}
V=\{1,2, \ldots, n\} & & \text { and } \\
E=C_{n} \cup D_{n}, & & \text { where }
\end{array}
$$

$C_{n}$ is a cycle of length $n, \quad C_{n}=\{(i, i+1) \mid i=1, \ldots, n\}$ and $D_{n}$ is the set of $s$-chords of $C_{n}, \quad D_{n}=\{(i, i+s) \mid i=1, \ldots, n\}$.

An $s$-chorded cycle graph gives rise to the definition of the corresponding $s$ chorded cycle inequality. In [Mut94b], it is investigated for which values of $s$, 
$t$ and $n$ the $s$-chorded cycle inequality induced by $G_{(n, s, t)}$ is valid, resp. facetdefining for $\mathcal{P} \mathcal{L S}\left(G_{(n, s, t)}\right)$.

Theorem 2 [Mut94b]. Let $G_{(n, s, t)}=(V, E)$ be a s-chorded cycle graph, i.e. $n=s t+r$ for $s, t, r \in \mathrm{N}$ and $r \in\{0,1, \ldots, s-1\}$. We define

$$
c_{e}= \begin{cases}n-2 t-s, & \text { if } x \in C_{n} \\ 1, & \text { if } x \in D_{n}\end{cases}
$$

and $c(E):=\sum_{e \in E} c_{e}$.

If $n-2 t-s>0$, the s-chorded cycle inequality

$$
(n-2 t-s) x\left(C_{n}\right)+x\left(D_{n}\right) \leq c(E)-(n-2 t)
$$

is valid for $\mathcal{P} \mathcal{L S}\left(G_{(n, s, t)}\right)$ if and only if $(t \geq 3, s \geq 3)$ or $(t=2, s \geq 3, r \geq 2)$. If the $s$-chorded cycle inequality is valid for $\mathcal{P} \mathcal{L} \mathcal{S}\left(G_{(n, s, t)}\right)$, then it is facetdefining for $\mathcal{P} \mathcal{L S}\left(G_{(n, s, t)}\right)$ if $r>0$.

Let $G_{(n, s, t)}$ be an s-chorded cycle graph, which is a subgraph of $G$. If $t=2$, or $s=3$, or $r \geq\left\lfloor\frac{s}{2}\right\rfloor$ and the corresponding $s$-chorded cycle inequality corresponding to $G_{(n, s, t)}$ is facet-defining for $\mathcal{P} \mathcal{L} \mathcal{S}\left(G_{(n, s, t)}\right)$, then the $s$-chorded cycle inequality is facet-defining for $\mathcal{P} \mathcal{L S}(G)$.

For $n=5$ the 2-chorded cycle inequality is identical to the Kuratowski inequality for $K_{5}$. So, the general $s$-chorded cycle inequalities are generalizations of the Kuratowski inequality for $K_{5}$. For the special case that $n=2 k+1$ and $s=k$, the $s$-chorded cycle graphs give rise to another kind of inequality, the odd $n$ ladder inequality, which is an alternative generalization of the $K_{5}$-inequality.

Theorem 3 [Mut94b]. If $G$ contains the $k$-chorded cycle graph $G_{(2 k+1, k, 2)}=$ $(V, E)$ on $2 k+1$ nodes with $E=C_{2 k+1} \cup D_{2 k+1}, k \in \mathrm{N}, k \geq 2$, then the odd $n$-ladder inequality

$$
(2 k-3) x\left(C_{2 k+1}\right)+x\left(D_{2 k+1}\right) \leq(2 k-1)^{2}
$$

is facet-defining for $\mathcal{P} \mathcal{L} \mathcal{S}\left(G_{(2 k+1, k, 2)}\right)$ and for $\mathcal{P} \mathcal{L} \mathcal{S}(G)$.

In the special case $s=k$ and $n=2 k$, the $k$-chorded cycle graphs $G_{(2 k, k, 2)}$ contain multiple edges. If we take each diagonal only once, we obtain a Möbius-ladder, which gives rise to an inequality which is a generalization of Kuratowski's $K_{3,3}$ inequality.

Definition 2 For $k \in \mathrm{N}, k \geq 3$, we define the (even) Möbius-ladder to be the graph $G_{M}=(V, E)$ with

$$
\begin{aligned}
& V=\{1,2, \ldots, 2 k\} \text { and } \\
& E=C_{2 k} \cup D_{k}, \quad \text { where }
\end{aligned}
$$

$C_{2 k}$ is a cycle of length $2 k, \quad C_{2 k}=\{(i, i+1) \mid i=1, \ldots, 2 k\}$,

$D_{k}$ is the set of longest chords of $C_{2 k}, \quad D_{k}=\{(i, i+k) \mid i=1,2, \ldots, k\}$. 
Theorem 4 [Mut94b]. For the Möbius-ladder graph $G_{M}=(V, E)$ with $E=$ $C_{2 k} \cup D_{k}$ and $k \geq 3$ the Möbius-ladder inequality

$$
(k-2) x\left(C_{2 k}\right)+x\left(D_{k}\right) \leq 2(k-1)^{2}
$$

is facet-defining for $\mathcal{P} \mathcal{L} S\left(G_{M}\right)$. Moreover, the Möbius ladder inequality is facetdefining for $\mathcal{P} \mathcal{L} \mathcal{S}(G)$ whenever $G_{M}$ is a subgraph of $G$.

A complete overview of the currently known structure of the planar subgraph polytope can be found in [Mut94a].

\section{The $k$-connected Subgraph Polytope $k-\mathcal{N C S}(G)$}

Given a $k$-connected graph $G=(V, E)$, we are interested in the set of all $k$ connected spanning subgraphs of $G$. For each $k$-connected spanning subgraph $K=(V, F)$ of $G$ we define its incidence vector $\chi^{F} \in \mathrm{R}^{E}$ by setting $\chi_{e}^{F}=1$ if $e \in F$ and $\chi_{e}^{F}=0$ if $e \notin F$. The $k$-connected (spanning) subgraph polytope $k-\mathcal{N} \mathcal{C S}(G)$ is defined as the convex hull over all incidence vectors of $k$-connected spanning subgraphs of $G$. In order to solve the minimum $k$-connected subgraph problem for a given $k$-connected graph $G=(V, E)$, we define the weight $w_{e}$ for an edge $e \in E$ to be 1 . The problem of determining a $k$-connected subgraph of $G$ with the minimum number of edges, can be formulated as the linear program $\min \left\{w^{T} x \mid x \in k-\mathcal{N} \mathcal{C S}(G)\right\}$. If we like to solve the $k$-augmentation problem for a given graph $G$, we choose $G_{0}=\left(V, E_{0}\right):=K_{n}$, define the weight $w_{e}$ for an edge to be $-M$, if $e \in E$ and 1 if $e \notin E$, where $M=\left|E_{0} \backslash E\right|+1$, and solve $\min \left\{w^{T} x \mid x \in k-\mathcal{N} \mathcal{C S}\left(G_{0}\right)\right\}$.

The $k$-connected subgraph polytope was already studied by Stoer in a more general form [Sto92]. For $k=2$, the computational results in [Sto92] are promising.

Theorem 5 [Sto92]. The integer points of $k-\mathcal{N} \mathcal{C S}(G)$ are characterized by the following system of inequalities:

$$
\begin{array}{ll}
0 \leq x_{e} \leq 1, & \text { for all } e \in E \\
x\left(\delta_{G-Y}(W)\right) \geq 1, & \text { for all } Y \subseteq V,|Y|=k-1, W \subseteq V \backslash Y \\
x_{e} \text { integral, } & \text { for all } e \in E
\end{array}
$$

The inequalities (5) essentially say that if a node set $Y \subseteq V$ of size $k-1$ is removed, the resulting graph must still be connected. 


\section{$4 \quad$ Intersecting $\mathcal{P} \mathcal{L S}(G)$ and $\boldsymbol{k}-\mathcal{N} \mathcal{C S}(G)$}

In this section we are interested in the integer points contained in the polytope $k-\mathcal{N} \mathcal{C P} \mathcal{L} \mathcal{S}(G)$ for $k=2$. They are identical to the integer points contained in the intersection of the polytopes $2-\mathcal{N} \mathcal{C S}(G)$ and $\mathcal{P} \mathcal{L S}(G)$. We already gave a system of inequalities characterizing the integer points for both polytopes. So, the integer points of the new polytope $k-\mathcal{N} \mathcal{C P} \mathcal{L S}(G)$ are defined by the system of inequalities given by (1), (2), (3) and (5).

One of the first questions occuring in connection to a polytope is its dimension. For general graphs $G$, the dimension of $2-\mathcal{N} \mathcal{C P} \mathcal{L} \mathcal{S}(G)$ is unlikely to be determined. Even for biconnected graphs $2-\mathcal{N} \mathcal{C P} \mathcal{L} S(G)$ may be empty, like, for example, for the Kuratowski graph shown in Figure 1(a). If we restrict our attention to $G=K_{n}$, the complete graph on $n$ nodes, we will see that $2-\mathcal{N} \mathcal{C P} \mathcal{L} \mathcal{S}\left(K_{n}\right)$ has full dimension.

Theorem 6 The dimension of the planar biconnected spanning subgraph polytope $2-\mathcal{N C P} \mathcal{L S}\left(K_{n}\right)$ for $K_{n}=(V, E)$, the complete graph on $n=|V|$ vertices, is $|E|$.

Proof. We will show that there are $|E|+1$ affinely independent elements in $R^{E}$ that are incidence vectors of planar, biconnected and spanning subgraphs of $K_{n}$. For simplicity let the vertices be numbered by $1,2, \ldots, n$. Let $P$ consist of the Hamiltonian cycle $C=((1,2),(2,3), \ldots,(n-1, n),(n, 1))$ together with the edge $e=(1,3)$. Adding any of the edges $e_{i} \in E \backslash(C \cup\{e\})$ gives $P^{i}, i=1,2, \ldots,|E|-$ $n-1$. Removing the edge $(u, u+1)$, resp. $(n, 1)$, and adding the edges $(u, v)$, $(v, u+1)$, resp. $(n, v),(v, 1)$, for any $v \notin[u-1 ; u+2]$ gives $P_{j}, j=1,2, \ldots, n$. Let $P^{\prime}$ be equal to $P \backslash\{(1,3)\}$. Then $P, P^{i}$ for $i=1,2, \ldots,|E|-n-1, P_{j}$ for $j=1,2, \ldots, n$, and $P^{\prime}$ induce planar, biconnected and spanning subgraphs of $K_{n}$. Moreover, all the $|E|+1$ incidence vectors $\chi^{P}, \chi^{P^{i}}$ for $i=1,2, \ldots,|E|-n-1$, $\chi^{P_{i}}$ for $j=1,2, \ldots, n$, and $\chi^{P^{\prime}}$ are linearly independent.

The natural question occurs whether known facets of the planar subgraph polytope are also facets for the planar biconnected spanning subgraph polytope. Suppose $c^{T} x \leq c_{0}$ is a facet of $\mathcal{P} \mathcal{L S}(G)$. It will also be a facet of $2-\mathcal{N} \mathcal{C P} \mathcal{L} \mathcal{S}(G)$ if $d=\operatorname{dim}(2-\mathcal{N} \mathcal{C P} \mathcal{L} \mathcal{S}(G))$ affinely independent incidence vectors of subgraphs $P \subseteq G$ exist that are planar, biconnected, spanning and satisfy the equation $c^{T} \chi^{P}=c_{0}$. The following theorem answers the question whether the support graph of a facet-defining inequality for $\mathcal{P} \mathcal{L S}\left(K_{n}\right)$ is biconnected. The support graph of an inequality $c^{T} x \leq c_{0}$, resp. $c^{T} x \geq c_{0}$, is induced by the edge set $\left\{e \in E \mid c_{e} \neq 0\right\}$.

Theorem 7 Let $c^{T} x \leq c_{0}$ be a facet of the planar subgraph polytope $\mathcal{P} \mathcal{L} S\left(K_{n}\right)$. Then either the inequality $c^{T} x \leq c_{0}$ is identical with $x_{e} \geq 0$ or $x_{e} \leq 1$, or the support graph of $c^{T} x \leq c_{0}$ is biconnected.

Proof. Let $S_{G}$ denote the edge set of the support graph of $c^{T} x \leq c_{0}$, and suppose that $G\left[S_{G}\right]$ is not biconnected with $\left|S_{G}\right| \geq 2$. Then, let $a$ be an articulation point 
of $S_{G}$ that separates the blocks induced by $K_{1}$ from $K_{2}:=S_{G} \backslash K_{1}$. At least one of the blocks induced by $K_{1}$ and $K_{2}$ is nonplanar (say $K_{1}$ ), since $S_{G}$ is nonplanar. We have $\left\{x \in \mathcal{P} \mathcal{L} \mathcal{S}(G) \mid c^{T} x=c_{0}\right\}=\left\{x \in \mathcal{P} \mathcal{L} \mathcal{S}\left(K_{n}\right) \mid c_{1}^{T} x+c_{2}^{T} x=c_{1}^{0}+c_{2}^{0}\right\}$, where $c_{i}$ denotes the vector of coefficients for block $K_{i}$ for $i=1,2$. We have that $\left\{x \in \mathcal{P} \mathcal{L} \mathcal{S}\left(K_{n}\right) \mid c^{T} x=c_{0}\right\} \subset\left\{x \in \mathcal{P} \mathcal{L} \mathcal{S}\left(K_{n}\right) \mid c_{1}^{T} x=c_{1}^{0}\right\}$, since otherwise we would have $c_{2}^{T} x>c_{2}^{0}$ or $c_{1}^{T} x>c_{1}^{0}$, both of which are nonvalid inequalities for $\mathcal{P} \mathcal{L} \mathcal{S}\left(K_{n}\right)$. Moreover, we have $\left\{x \in \mathcal{P} \mathcal{L} \mathcal{S}\left(K_{n}\right) \mid c^{T} x=c_{0}\right\} \neq\left\{x \in \mathcal{P} \mathcal{L} \mathcal{S}\left(K_{n}\right) \mid\right.$ $\left.c_{1}^{T} x=c_{1}^{0}\right\}$. Hence the inequality $c^{T} x \leq c_{0}$ can not be facet-defining for $\mathcal{P} \mathcal{L} \mathcal{S}\left(K_{n}\right)$, which is a contradiction.

In general, biconnectivity of the support graph of a facet-defining inequality $c^{T} x \leq c_{0}$ for $\mathcal{P} \mathcal{L S}(G)$ is not sufficient to guarantee that it is also a facet for $2-\mathcal{N} \mathcal{C} \mathcal{P} \mathcal{L} \mathcal{S}(G)$. Consider, for example, the subdivision $S$ of $K_{5}$ shown in Figure 1(a). The Kuratowski inequalities that are facets for $\mathcal{P} \mathcal{L} \mathcal{S}(S)$, are not facets for $2-\mathcal{N} \mathcal{C} \mathcal{P} \mathcal{L} S(S)$, since removing any edge of $S$ leads to a graph that is not biconnected. Fortunately, for complete graphs the situation is promising. We can show the following theorem.

Theorem 8 Let (7) $c^{T} x \leq c_{0}$ be a facet of the planar subgraph polytope $\mathcal{P} \mathcal{L} \mathcal{S}\left(K_{n}\right)$. Then $c^{T} x \leq c_{0}$ is also a facet of the planar, biconnected and spanning subgraph polytope $2-\overline{\mathcal{N}} \mathcal{C P} \mathcal{L} \mathcal{S}\left(K_{n}\right)$.

Proof. Let $K_{n}=(V, E)$. From Theorem 7 we know that either the inequality $c^{T} x \leq c_{0}$ is identical with $x_{e} \geq 0$ or $x_{e} \leq 1$, or the support graph of $c^{T} x \leq c_{0}$ is biconnected. For the inequalities $x_{e} \geq 0$ and $x_{e} \leq 1$ we give a direct proof similar to the one for Theorem 6 . For simplicity the nodes are numbered by $1,2, \ldots, n$. We first show that $x_{e} \leq 1$ is a facet-defining inequality for $2-\mathcal{N} \mathcal{C P} \mathcal{L} \mathcal{S}\left(K_{n}\right)$. Without any restriction let $e=(1,3)$. Consider the graphs induced by the edge sets $P, P^{i}$ for $i=1,2, \ldots|E|-n-1$ and $P_{j}$ for $j=1,2, \ldots, n$ defined in the proof to Theorem 6 . All of them are planar, biconnected and spanning, satisfy $x_{e}=1$ and their incidence vectors are linearly independent. Next, removing the edge $e=(1,3)$ from $P, P^{i}$ for $i=1,2, \ldots|E|-n-1$ and $P_{j}$ for $j=1,2, \ldots, n$ gives us still $|E|$ planar, biconnected and spanning subgraphs of $K_{n}$, satisfying $x_{e}=0$. Moreover, their incidence vectors are affinely independent.

Now we will show the facet-defining properties of those inequalities $c^{T} x \leq c_{0}$ which are facet-defining for $\mathcal{P} \mathcal{L} \mathcal{S}\left(K_{n}\right)$ and have biconnected support. There are sufficiently many planar subgraphs satisfying (7) with equality that can be used in an indirect proof to show the facet-defining property of (7) for $\mathcal{P} \mathcal{L} \mathcal{S}\left(K_{n}\right)$. That is, suppose there exists an inequality $a^{T} x \leq a_{0}$ with $\left\{x \in \mathcal{P} \mathcal{L S}\left(K_{n}\right)\right.$ $\left.c^{T} x=c_{0}\right\} \subseteq\left\{x \in \mathcal{P} \mathcal{L} \mathcal{S}\left(K_{n}\right) \mid a^{T} x=a_{0}\right\}$. From this it follows that $c^{T}=a^{T}$ and $c_{0}=a_{0}$. If all these planar subgraphs are biconnected and spanning, we have already found enough affinely independent points of $2-\mathcal{N} \mathcal{C P} \mathcal{L} S\left(K_{n}\right)$ in $\left\{x \in 2-\mathcal{N} \mathcal{C P} \mathcal{L} \mathcal{S}\left(K_{n}\right) \mid c^{T} x=c_{0}\right\}$. Suppose, some of these subgraphs are not biconnected and spanning. Then we claim that we can augment them to planar, biconnected and spanning subgraphs of $K_{n}$ by adding a set $F \subseteq E$, with $c(F)=0$ and $a(F)=0$. With the new set of independent points in $\left\{x \in 2-\mathcal{N} \mathcal{C P} \mathcal{L} \mathcal{S}\left(K_{n}\right) \mid\right.$ 
$\left.c^{T} x=c_{0}\right\}$ we can proceed as in the indirect proof for the facet-defining property of (7) for $\mathcal{P} \mathcal{L} \mathcal{S}(G)$.

Let $P$ be the edge set of a planar subgraph used in the indirect proof for $\mathcal{P} \mathcal{L} \mathcal{S}\left(K_{n}\right)$, that is not biconnected nor spanning with $c^{T} \chi^{P}=c_{0}$. Let $a$ be an articulation point of $P$ separating the blocks induced by $K_{1}$ from $K_{2}:=P \backslash K_{1}$. Let $v_{i}$ be a vertex next to $a$ at the outer face of a planar embedding of block $K_{i}$, $i=1,2$. Adding the edge $e=\left(v_{1}, v_{2}\right)$ to $P$ still maintains a planar subgraph $P_{1}$, where $a$ does not anymore separate component $K_{1}$ from $K_{2}$. Moreover, we have $c_{0} \geq c^{T} \chi^{P_{1}}=c^{T} \chi^{P}+c_{e}=c_{0}+c_{e}$, hence $c_{e}=0$ that implies $a_{e}=0$. Repeating this iteration subsequently for all articulation points in $P$ leads to a biconnected planar subgraph $P_{k}$ with $P_{k}=P \cup F$ with $c(F)=0$ and $a(F)=0$. If $P_{k}$ is not spanning, we add the edges $(z, u),(z, w)$ for $(u, w) \in P_{k}$ and all vertices $z$ that are not contained in $P_{k}$. Now the new edge set $P_{k}^{\prime}=P_{k} \cup F^{\prime}$ is spanning, while planarity and biconnectivity is maintained. Using the same arguments as before gives $c\left(F^{\prime}\right)=a\left(F^{\prime}\right)=0$.

Our investigations concerning the facet-defining inequalities arising from biconnectivity conditions lead to the following theorem. For $k=2$, inequalities (5) reduce to the ones given in Theorem 9 .

Theorem 9 Let $K_{n}=(V, E)$ be the complete graph on $n$ nodes. Furthermore, let $z \in V$, and a set $W \subseteq V \backslash\{z\}$ with $\emptyset \neq W \neq V \backslash\{z\}$. The node-cut constraint

$$
x\left(\delta_{G-\{z\}}(W)\right) \geq 1
$$

defines a facet of $2-\mathcal{N} \mathcal{C P} \mathcal{L S}\left(K_{n}\right)$.

Proof. We will prove it indirect. Let $c^{T} x \geq c_{0}$ denote inequality (8). Suppose there exists an inequality $a^{T} x \geq a_{0}$ with $\left\{x \in 2-\mathcal{N} \mathcal{C P} \mathcal{L} \mathcal{S}\left(K_{n}\right) \mid c^{T} x=c_{0}\right\} \subseteq$ $\left\{x \in 2-\mathcal{N} \mathcal{C P} \mathcal{L S}\left(K_{n}\right) \mid a^{T} x=a_{0}\right\}$. We will show that then $c^{T}=\lambda a^{T}$ and $c_{0}=$ $\lambda a_{0}$ for $\lambda>0$. For any fixed $z \in V$ and fixed set $W \subseteq V \backslash\{z\}, \emptyset \neq W \neq V \backslash\{z\}$ let $U=V \backslash\{W \cup z\}$. We will construct a planar, biconnected and spanning subgraph of $G$ induced by the edge set $P \subseteq E$. $P$ consists of the edge sets of an Hamiltonian cycle $H_{W}$ in $W$ and $H_{U}$ in $U$, resp. an Hamiltonian Path $H_{W}$ if $|W|=2$ or $H_{U}$ if $|U|=2$, the edge $(u, w)$ for $u \in U, w \in W$, and the edges $(u, z)$, $\left(u^{\prime}, z\right),(z, w)$ and $\left(z, w^{\prime}\right)$ for $u \neq u^{\prime} \in U$, if $|U| \geq 2, w \neq w^{\prime} \in W$ if $|W| \geq 2$. Obviously, $P$ is the edge set of a planar, biconnected and spanning subgraph of $G$ satisfying inequality (8) by equality, hence also $a^{T} \chi^{P}=a_{0}$.

Substituting in $P$ the edge $(u, w)$ by any of the edges $\left(u^{\prime \prime}, w^{\prime \prime}\right), u^{\prime \prime} \in U, w^{\prime \prime} \in$ $W$ still yields a planar, biconnected spanning subgraph $P_{1}$ satisfying $c^{T} \chi^{P_{1}} \geq c_{0}$ with equality, hence also $a^{T} \chi^{P_{1}}=a_{0}$. We have $0=c^{T} \chi^{P}-c^{T} \chi^{P_{1}}=a^{T} \chi^{P}{ }_{-}$ $a^{T} \chi^{P_{1}}=a_{(u, w)}-a_{\left(u^{\prime \prime}, w^{\prime \prime}\right)}$, hence $a_{(u, w)}=a_{\left(u^{\prime \prime}, w^{\prime \prime}\right)}$ for all $u, u^{\prime \prime} \in U, w, w^{\prime \prime} \in W$.

By adding any edge contained in the complete graph induced by $W$ to $H_{W}$ the required properties are still satisfied, hence $a_{e}=0$ for all $e \in G\left[W \backslash H_{W}\right]$. If $|W| \geq 4$, then by choosing a different Hamiltonian cycle in $G[W]$, we get $a_{e}=0$ for all $e \in G[W]$. In the case that $|W|=3$, we can remove the edge $e=\left(w, w^{\prime}\right)$ 
from $P$ without loosing biconnectivity, hence we get $a_{e}=0$ for all $e \in G[W]$. The same arguments hold for the set $U$, so we have $a_{e}=0$ for all $e \in G[U]$.

Moreover, adding any of the edges $\left(z, w^{\prime \prime}\right), w^{\prime \prime} \in W \backslash\left\{w, w^{\prime}\right\}$ to $P$ still yields a planar, biconnected spanning subgraph of $G$ satisfying inequality (8) with equality. Hence $a_{(z, w)}=0$ for $w \in W$ and, because of symmetry reasons, $a_{(z, u)}=0$ for $u \in U$. Hence we have shown $a_{e}=\lambda c_{e}$, and $a_{0}=\lambda c_{0}$ for all $e \in E$.

\section{$5 \quad$ Algorithm}

In [JM94] we give a branch and cut algorithm for the maximum planar subgraph problem using facet-defining inequalities for $\mathcal{P} \mathcal{L} \mathcal{S}(G)$ as cutting planes. We have to change and to add only a few routines in order to get a branch and cut algorithm for solving the three problems, the minimum planarizing 2augmentation problem, the planar 2-augmentation problem and the maximum planar biconnected spanning subgraph problem.

In a cutting plane algorithm, a sequence of relaxations is solved by linear programming. After the solution $x$ of some relaxation is found, we must be able to check whether $x$ is the incidence vector of a planar, biconnected and spanning subgraph (in which case we have solved the problem) or whether any of the known facet-defining inequalities are violated by $x$. If no such inequalities can be found, we cannot tighten the relaxation and have to resort to branching, otherwise we tighten the relaxation by all facet-defining inequalities violated by $x$ which we can find. Then the new relaxation is solved, etc. The process of finding violated inequalities (if possible) is called "separation" or "cutting plane generation".

The cutting plane generation as well as the lower bound heuristic for the planarity part are based on a planarity testing algorithm of Hopcroft and Tarjan [HT74]. At the beginning we solve the Linear Program (LP) consisting of the trivial inequalities $x_{e} \geq 0, x_{e} \leq 1$ and the inequality $x(E) \leq 3|V|-6$. Let $x$ be an LP-solution produced in the cutting plane procedure applied in some node of the enumeration tree. For $0 \leq \varepsilon \leq 1$ we define $E_{\varepsilon}=\left\{e \in E \mid x_{e} \geq 1-\varepsilon\right\}$ and consider $G_{\varepsilon}=\left(V, E_{\varepsilon}\right)$. For the unweighted graph $G_{\varepsilon}$ the linear planarity testing algorithm of Hopcroft and Tarjan is called. The algorithm stops if it finds an edge set $F$ which is not planar. In case the inequality $x(F) \leq|F|-1$ is violated, we reduce it to a facet-defining inequality before we add it to the constraints of the current LP. We also use a heuristic which searches for violated Euler-inequalities and inequalities given by some classes of $s$-chorded cycle graphs.

The cutting plane generation for achieving biconnectivity makes use of the facet-defining inequalities given in (5). For $k=2$, the inequalities reduce to the ones given in (8). Given an LP-solution $x$ produced in the cutting plane procedure, we are able to give a node $z \in V$ and a set $W, \emptyset \neq W \subset V \backslash\{z\}$ violating inequality (8) or guaranteeing that all the inequalities in (8) are satisfied by $x$. This can be done in polynomial time by the following separation routine. For all nodes $z \in V$ construct the graph $G^{\prime}:=G-\{z\}=\left(V^{\prime}, E^{\prime}\right)$ and search for 


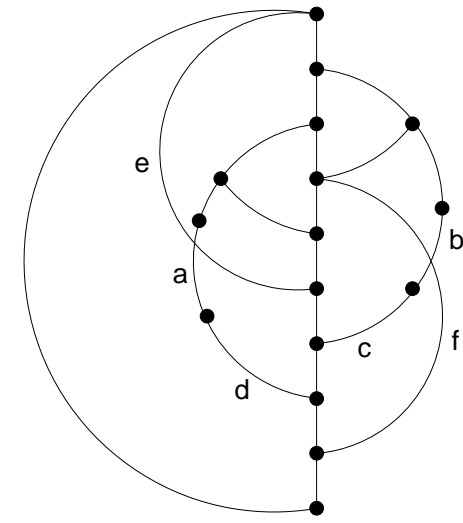

(a)

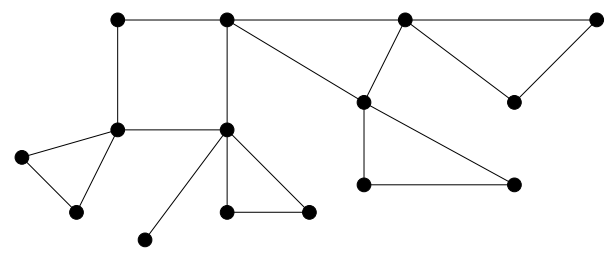

(b)

Fig. 3. shows two of the instances used for the computations. For the graph shown in (a) the approach, first to determine a maximum planar subgraph may lead to removing the edges $a$ and $b$. In order to augment the graph to biconnectivity, we have to add at least three edges, whereas the optimum solution of the minimum planarizing 2-augmentation problem removes the edges $e$ and $f$. The graph shown in (b) has been given by Kant. The heuristic he suggests adds 5 new edges to the graph in order to obtain a planar biconnected graph, whereas the optimum solution is to add $\mathbf{3}$ edges.

the minimum cut in $G^{\prime}$ with edge values $x_{e}$ for all $e \in E^{\prime}$. Let $y$ be the value of this minimum cut. If $y \geq 1$, all inequalities of the type (8) for the specific node $z$ are satisfied. Otherwise, the inequality $x\left(\delta_{G-\{z\}}(W)\right) \geq 1$ is violated for the set $W$ determined by the minimum cut $\delta(W)$.

Although the vectors $x$ coming up as solutions of LP-relaxations in the above outlined process have fractional components in general, they are often useful to obtain information on how a high-valued planar subgraph might look like. We exploit this idea with a greedy type heuristic with respect to the solution values of the edges. Starting from the empty graph, a planar subgraph is constructed by adding the edges in order of decreasing values if they do not destroy planarity. In a second phase this planar subgraph is augmented to a biconnected, spanning and planar subgraph by a trivial heuristic. So, in addition to the upper bounds $w^{T} x$ on the value of a maximum planar biconnected spanning subgraph, we also obtain a lower bound $w^{T} \bar{x}$ from the incidence vector $\bar{x}$ of the planar, biconnected and spanning subgraph derived heuristically from $x$.

\section{Computational Experiments}

The computational experiments were run on a Solbourne 5E/702. For our "first" experiments we used the above described algorithm. Our current preliminary implementation does not yet contain a procedure that tries to find good feasible solutions whose objective function values constitute lower bounds. However, in all examples discussed here, the optimum solution was found by cutting planes, i.e. the LP-solution $x$ turned out to be the incidence vector of a planar, biconnected and spanning subgraph. 
Table 1. Computational Results for graphs from the literature

\begin{tabular}{|r|r|r|r|r|r|r|r|r|r|r|r|}
\hline Problemname & $|V|$ & $|E|$ & \#Var & Del1 & Add1 & Tim1 & Del2 & Add2 & Tim2 & Del3 & Tim3 \\
\hline Jaya.10.22 & 10 & 22 & 45 & 2 & 0 & 1 & 2 & 0 & 0 & 2 & 1 \\
Beck.11.21 & 11 & 21 & 55 & 1 & 1 & 0 & 1 & 1 & 0 & - & - \\
JueMut.14.20 & 14 & 20 & 91 & 2 & 0 & 1 & 2 & 0 & 0 & 2 & 1 \\
JueMut.16.22 & 16 & 22 & 120 & 2 & 0 & 1 & 2 & 3 & 2 & 2 & 1 \\
Kant.15.20 & 15 & 20 & 105 & 0 & 3 & 0 & 0 & 3 & 0 & - & - \\
EadMar.20.30 & 20 & 30 & 190 & 2 & 0 & 10 & 2 & 0 & 1 & 2 & 10 \\
Harel.20.35 & 20 & 35 & 190 & 3 & 0 & 11 & 2 & 1 & 0 & 3 & 4 \\
Martin.30.56 & 30 & 56 & 435 & 3 & 3 & 19 & 3 & 3 & 6 & - & - \\
Hims.34.45 & 34 & 45 & 561 & 2 & 6 & 335 & 2 & 6 & 192 & - & - \\
Kant.45.85 & 45 & 85 & 990 & 3 & 0 & 231 & 3 & 0 & 15 & 3 & 421 \\
Hims.46.64 & 46 & 64 & 1035 & 2 & 1 & 64 & 2 & 2 & 23 & - & - \\
Cim.60.166 & 60 & 160 & 1770 & 1 & 0 & 17 & 1 & 0 & 17 & 1 & 32 \\
\hline
\end{tabular}

Table 1 shows computational results for some graphs in the [LAYOUT-LIB], a library for benchmark sets in Automatic Graph Drawing. The columns show the problem name, the number of nodes and edges of the given graph $G$, and the number of variables. Moreover, it shows the solution of the minimum planarizing 2-augmentation problem, i.e. the number of edges removed from $G$ and added to $G$, and the solution time in seconds. Columns $8-10$ show the solution, when first a maximum planar subgraph is determined and then the planar 2-augmentation problem is been solved. Surprisingly, the total time for solving both problems, the maximum planar subgraph problem and the planar 2-augmentation problem, is in many cases much less than the time for solving the minimum planarizing 2 -augmentation problem. Moreover, the solutions found by the two different methods are in most cases identical. We do not have a code for augmenting a nonplanar graph to biconnectivity. Hence, we only give the results of the third approach, first augmenting the graph to biconnectivity and then solving the maximum planar biconnected subgraph problem, for the graphs that are already biconnected.

Consider the graph shown in Figure 3 (JueMut.16.22). In the run for solving the minimum planarizing 2-augmentation problem, 14 violated node-cut constraints have been found, 14 Kuratowski-inequalities and 1 Euler-inequality. It took 17 LP's with 4 branch and cut nodes in 1.48 seconds. At the end, the two edges $e$ and $f$ have been deleted. This solution is identical to the solution for the maximum planar biconnected subgraph problem. Solving first the maximum planar subgraph problem leads to removing the edges $a$ and $b$ (using 10 Kuratowski inequalities and 1 Euler inequality, 6 LP's in the root node in 0.54 seconds). Solving the planar 2-augmentation problem on the obtained planar subgraph leads to adding three new edges. In this case 8 violated node-cut inequalities were detected, 34 Kuratowski inequalities and 1 Euler inequality. The computation took 31 LP's and 8 branching nodes in 2.33 seconds.

This is the first time that these problems have been solved to optimality. We think that we will be able to solve even bigger instances in the near future. 


\section{References}

[ET76] Eswaran, K.P., and R.E. Tarjan: Augmentation problems. SIAM Journal on Computing 5 (1976) 653-665

[Fra92] Frank, A.: Augmenting graphs to meet edge-connectivity requirements. SIAM J. Discr. Math. 5 (1992) 25-53

[GJ79] Garey, M.R., and D.S. Johnson: Computers and Intractability: A Guide to the Theory of NP-completeness. Freeman, San Franc. (1979)

[GSS93] Garg, N., V.S. Santosh, and A. Singla: Improved Approximation Algorithms for Biconnected Subgraphs via Better Lower Bounding Techniques. SODA (1993), 103-111

[GT94] Goldschmidt, O. and A. Takvorian: Finding a Biconnected Spanning Planar Subgraph of a Nonplanar Graph. Technical Report ORP93-03, University of Texas, Austin, USA (1994)

[Har62] Harary, F.: The maximum connectivity of a graph Proceed. of the National Academy of Sciences. USA 48 (1962) 1142-1146

[Him93] Himsolt, M.: Konzeption und Implementierung von Grapheneditoren. Dissertation, Universität Passau (1993)

[HT74] Hopcroft, J., and R.E. Tarjan: Efficient planarity testing. J. ACM 21 (1974) 549-568

[JM94] Jünger, M. and P. Mutzel: Maximum planar subgraphs and nice embeddings: Practical layout tools. to appear in Algorithmica, special issue on Graph Drawing, Ed. G. Di Battista und R. Tamassia (1994)

[JM95] Jünger, M. and P. Mutzel: The Polyhedral Approach to the Maximum Planar Subgraph Problem: New Chances for Related Problems. Lect. Notes in Comp. Sci 894, Proc. DIMACS GD'94, Ed. R. Tamassia and I.G. Tollis, Springer-Verlag, Princeton (1995) 119-130

[HR91] Hsu, T.-S. and V. Ramachandran: A linear time algorithm for triconnectivity augmentation. Proc. 32th Annual Symp. on Found. of Comp. Science, Puerto Rico (1991) 548-559

[Kan93] Kant, G.: Algorithms for Drawing Planar Graphs. Ph.D.-Thesis, Utrecht University (1993)

[LAYOUT-LIB] per ftp available at layoutlib@informatik.uni-koeln.de (1995)

[Mut94a] Mutzel, P.: The Maximum Planar Subgraph Problem. Dissertation, Universität Köln (1994)

[Mut94b] Mutzel, P.: s-Chorded Cycle Graphs and their Relation to the Planar Subgraph Polytope. Technical Report No. 94-161, Angewandte Mathematik und Informatik, Universität zu Köln (1994)

[RW 95] Ravi, R. and D.P. Williamson: An Approximation Algorithm for Minimum-Cost Vertex-Connectivity Problems. Proc. 6th Annual ACM-SIAM Symposium on Discrete Algorithms, San Francisco (1995)

[RG77] Rosenthal, A. and A. Goldner: Smallest augmentation to biconnect a graph. SIAM J. on Computing 6 (1977) 55-66

[Sto92] Stoer, M.: Design of Survivable Networks. Lecture Notes in Mathematics, Springer-Verlag, Berlin (1992)

[TBB88] Tamassia, R., G. Di Battista, and C. Batini: Automatic graph drawing and readability of diagrams. IEEE Transactions on Systems, Man and Cybernetics 18 (1988) 61-79

[WN93] Watanabe, T., and A. Nakamura: A minimum 3-connectivity augmentation of a graph. J. Comp. and Sys. Sci. 46 (1993) 91-128 
This article was processed by the author using the $\mathrm{T}_{\mathrm{E}} \mathrm{X}$ macro package from SpringerVerlag. 